\title{
Stepwise Precession of the Resonant Swinging Spring
}

\author{
Darryl D. Holm* \\ Theoretical Division and CNLS \\ Los Alamos National Laboratory, MS B284 \\ Los Alamos, NM 87545 \\ Peter Lynch $^{\dagger}$ \\ Met Éireann \\ Glasnevin Hill \\ Dublin 9, Ireland
}

\begin{abstract}
The swinging spring, or elastic pendulum, has a 2:1:1 resonance arising at cubic order in its approximate Lagrangian. The corresponding modulation equations are the wellknown three-wave equations that also apply, for example, in laser-matter interaction in a cavity. We use Hamiltonian reduction and pattern evocation techniques to derive a formula that describes the characteristic feature of this system's dynamics, namely, the stepwise precession of its azimuthal angle.
\end{abstract}

PACS numbers: 02.40.-k, 05.45.-a, 45.10.Db, 45.20.Jj

Keywords: Classical mechanics, Variational principles, Averaged Lagrangian, Elastic Pendulum, Nonlinear Resonance.

*email: dholm@lanl.gov

${ }^{\dagger}$ email: Peter.Lynch@met.ie 


\section{Introduction}

\subsection{Problem statement, approach and summary of results}

The elastic pendulum or swinging spring is a simple mechanical system that exhibits complex dynamics. It consists of a heavy mass suspended from a fixed point by a light spring which can stretch but not bend, moving under gravity. We investigate the 2:1:1 resonance dynamics of this system in three dimensions and study its characteristic feature - the regular stepwise precession of its azimuthal angle.

When the Lagrangian is approximated to cubic order and averaged over the fast dynamics, the resulting modulation equations have three independent constants of motion and are completely integrable. These modulation equations are identical to the three-wave equations for resonant triad interactions in fluids and plasmas, and in laser-matter interaction. We reduce the system to a form amenable to analytical solution and show how the full solution may be reconstructed. We examine the geometry of the solutions in phase-space and develop a number of simple qualitative descriptions of the motion.

We compare solutions of the exact and modulation equations and show that they are remarkably similar. A characteristic stepwise precession occurs as the motion cycles between quasi-vertical and quasi-horizontal. That is, during each quasi-vertical phase, the azimuth of the swing plane precesses by a constant angular increment. This stepwise azimuthal precession occurs in bursts, when the motion is nearly vertical. By transforming to non-uniformly rotating coordinates and assuming a geometric constraint (essentially the method of pattern evocation), we find a formula for the rotation of the swing plane. This formula gives a highly accurate description of the stepwise precession of the azimuthal angle of the motion.

\subsection{History of the problem}

The first comprehensive analysis of the elastic pendulum appeared in Vitt and Gorelik (1933). These authors were inspired by the analogy between this system and the Fermi resonance of a carbon-dioxide molecule. We make connections in this paper with other physical systems of current interest. For example, we show that the modulation equations for the averaged motion of the swinging spring may be transformed into the equations for three-wave interactions that appear in analyzing fluid and plasma systems, and in laser-matter interaction. These three complex equations are also identical to the Maxwell-Schrödinger envelope equations for the interaction between radiation and a two-level resonant medium in a microwave cavity (Holm and Kovačič, 1992). The three-wave equations also govern the envelope dynamics of light waves in an inhomogeneous material (David et al., 1990, Alber et al., 1998a,b). For the special case where the Hamiltonian takes the value zero, the equations reduce to Euler's equations for a freely rotating rigid body. Finally, the equations are also equivalent to a complex (unforced and undamped) version of the Lorenz (1963) three-component model, which has been the subject of many studies (Sparrow, 1982). Thus, the simple spring pendulum, which was first studied to provide a classical analogue to 
the quantum phenomenon of Fermi resonance, now provides a concrete mechanical system which simulates a wide range of physical phenomena.

All of the previous studies of the spring pendulum known to us have considered motion in two dimensions. To our knowledge, only Cayton (1977) discussed threedimensional solutions and observed the curious rotation of the swing plane between successive cycles when the horizontal energy is maximum. This particular aspect of the behavior of the swinging spring in three dimensions is its most striking difference from two-dimensional motions. Suppose the system is excited initially near its vertical oscillation mode. Since purely vertical motion is unstable, horizontal motion soon develops. The horizontal oscillations grow to a maximum and then subside again. An alternating cycle of quasi-vertical and quasi-horizontal oscillations recurs indefinitely. Seen from above, during each horizontal excursion of several oscillations the projected motion is approximately elliptical. Experimentally and numerically one observes that between any two successive horizontal excursions the orientation of the projected ellipse rotates by the same angle, thereby causing a stepwise precession of the swing plane. In principle, the precession angle between successive horizontal excursions can be deduced from the complete solution of the integrable envelope equations. We seek a simple approximate expression for the precession of the swing plane in terms of the solution of the reduced dynamics.

Lynch (2001) found a particular solution for the rate of precession of the swing plane by using the method of multiple time scales in rotating coordinates and introducing a certain angular solution Ansatz. We recover Lynch's particular solution among a family of other solutions for the swing plane precession rate. This family is obtained via the method of averaged Lagrangians by seeking solutions of the modulation equations that satisfy a geometrical constraint of being "instantaneously elliptical." We apply the method of pattern evocation in shape space (Marsden, et al., 1995, 1996). Using this process, one identifies patterns by viewing the dynamics relative to rotating frames with certain critical angular velocities. Our numerical integrations show that the solution resulting from this geometrical postulate estimates the precession of the swing plane with surprisingly high accuracy.

\section{Equations of motion}

The physical system under investigation is an elastic pendulum, or swinging spring, consisting of a heavy mass suspended from a fixed point by a light spring which can stretch but not bend, moving under gravity, $g$. We assume an unstretched length $\ell_{0}$, length $\ell$ at equilibrium, spring constant $k$ and unit mass $m=1$. The corresponding Lagrangian, approximated to cubic order in the amplitudes, is

$$
L=\frac{1}{2}\left(\dot{x}^{2}+\dot{y}^{2}+\dot{z}^{2}\right)-\frac{1}{2}\left(\omega_{R}^{2}\left(x^{2}+y^{2}\right)+\omega_{Z}^{2} z^{2}\right)+\frac{1}{2} \lambda\left(x^{2}+y^{2}\right) z,
$$

where $x, y$ and $z$ are Cartesian coordinates centered at the point of equilibrium, $\omega_{R}=\sqrt{g / \ell}$ is the frequency of linear pendular motion, $\omega_{Z}=\sqrt{k / m}$ is the frequency of its elastic oscillations and $\lambda=\ell_{0} \omega_{Z}^{2} / \ell^{2}$. The system is illustrated schematically in 
Fig. 1. The Euler-Lagrange equations of motion may be written

$$
\begin{aligned}
\ddot{x}+\omega_{R}^{2} x & =\lambda x z, \\
\ddot{y}+\omega_{R}^{2} y & =\lambda y z, \\
\ddot{z}+\omega_{Z}^{2} z & =\frac{1}{2} \lambda\left(x^{2}+y^{2}\right) .
\end{aligned}
$$

There are two constants of the motion, the total energy $E$ and the angular momentum $h$ given by

$$
E=\frac{1}{2}\left(\dot{x}^{2}+\dot{y}^{2}+\dot{z}^{2}\right)+\frac{1}{2}\left(\omega_{R}^{2}\left(x^{2}+y^{2}\right)+\omega_{Z}^{2} z^{2}\right)-\frac{1}{2} \lambda\left(x^{2}+y^{2}\right) z, \quad h=(x \dot{y}-y \dot{x}) .
$$

The system is not integrable. Its chaotic motions have been studied by many authors (see, e.g., Refs. in Lynch, 2001). Previous studies have considered the two dimensional case, for which the angular momentum $h$ vanishes.

We confine attention to the resonant case $\omega_{Z}=2 \omega_{R}$ and apply the averaged Lagrangian technique (Whitham, 1974). The solution of (2.2)-(2.4) is assumed to be of the form

$$
\begin{aligned}
& x=\Re\left[a_{0}(t) \exp \left(i \omega_{R} t\right)\right], \\
& y=\Re\left[b_{0}(t) \exp \left(i \omega_{R} t\right)\right], \\
& z=\Re\left[c_{0}(t) \exp \left(2 i \omega_{R} t\right)\right] .
\end{aligned}
$$

(The zero-subscripts in $a_{0}, b_{0}$ and $c_{0}$ are introduced to distinguish from the variables $a, b$ and $c$ in a rotating frame, introduced below.) The coefficients $a_{0}(t), b_{0}(t)$ and $c_{0}(t)$ are assumed to vary on a time scale which is much longer than the time-scale of the oscillations, $\tau=1 / \omega_{R}$. The Lagrangian is averaged over time $\tau$ to give,

$$
\langle L\rangle=\frac{1}{2} \omega_{R}\left[\Im\left\{\dot{a}_{0} a_{0}^{*}+\dot{b}_{0} b_{0}^{*}+2 \dot{c}_{0} c_{0}^{*}\right\}+\kappa \Re\left\{\left(a_{0}^{2}+b_{0}^{2}\right) c_{0}^{*}\right\}\right],
$$

where $\kappa=\lambda /\left(4 \omega_{R}\right)$. We regard the quantities $a_{0}, b_{0}, c_{0}$ as generalized coordinates. The averaged Lagrangian equations of motion are then

$$
\begin{aligned}
i \dot{a}_{0} & =\kappa a_{0}^{*} c_{0}, \\
i \dot{b}_{0} & =\kappa b_{0}^{*} c_{0}, \\
i \dot{c}_{0} & =\frac{1}{4} \kappa\left(a_{0}^{2}+b_{0}^{2}\right)
\end{aligned}
$$

Eqns. (2.8)-(2.10) are the complex versions of Eqns. (68)-(73) in Lynch (2001), which were derived using the method of multiple time-scale analysis.

We now absorb the constant $\kappa$ by rescaling the time, $t \rightarrow \kappa t$, and transform variables

$$
A=\frac{1}{2}\left(a_{0}+i b_{0}\right), \quad B=\frac{1}{2}\left(a_{0}-i b_{0}\right), \quad C=c_{0} .
$$

Consequently, the equations of motion take the form

$$
\begin{aligned}
i \dot{A} & =B^{*} C, \\
i \dot{B} & =C A^{*}, \\
i \dot{C} & =A B .
\end{aligned}
$$


These three complex equations are well-known as the three-wave interaction equations, which govern quadratic wave resonance in fluids and plasmas.

The three-wave interaction equations (2.11)-(2.13) may be written in canonical form with Hamiltonian $H=\Re\left(A B C^{*}\right)$ and Poisson brackets $\left\{A, A^{*}\right\}=\left\{B, B^{*}\right\}=$ $\left\{C, C^{*}\right\}=-2 i$, as

$$
\begin{aligned}
i \dot{A} & =i\{A, H\}=2 \partial H / \partial A^{*}, \\
i \dot{B} & =i\{B, H\}=2 \partial H / \partial B^{*}, \\
i \dot{C} & =i\{C, H\}=2 \partial H / \partial C^{*}
\end{aligned}
$$

These equations conserve the following three quantities,

$$
\begin{aligned}
H & =\frac{1}{2}\left(A B C^{*}+A^{*} B^{*} C\right)=\Re\left(A B C^{*}\right), \\
N & =|A|^{2}+|B|^{2}+2|C|^{2}, \\
J & =|A|^{2}-|B|^{2} .
\end{aligned}
$$

Thus, the modulation equations for the swinging spring are transformed into the three-wave equations, which are known to be completely integrable. See Alber et al. (1998a) for references to the three wave equations and an extensive elaboration of their properties as a paradigm for Hamiltonian reduction.

The following positive-definite combinations of $N$ and $J$ are physically significant:

$$
N_{+} \equiv \frac{1}{2}(N+J)=|A|^{2}+|C|^{2}, \quad N_{-} \equiv \frac{1}{2}(N-J)=|B|^{2}+|C|^{2} .
$$

These combinations are known as the Manley-Rowe relations in the extensive literature about three-wave interactions. The quantities $H, N_{+}$and $N_{-}$provide three independent constants of the motion.

\subsection{A brief history of the three-wave equations}

Fluids and plasmas. The three-wave equations model the nonlinear dynamics of the amplitudes of three waves in fluids or plasmas (Bretherton, 1964). The equations result from a perturbation analysis of the Charney equation

$$
\frac{\partial}{\partial t}\left[\nabla^{2} \psi-F \psi\right]+\left(\frac{\partial \psi}{\partial x} \frac{\partial \nabla^{2} \psi}{\partial y}-\frac{\partial \psi}{\partial y} \frac{\partial \nabla^{2} \psi}{\partial x}\right)+\beta \frac{\partial \psi}{\partial x}=0
$$

(see, e.g., Pedlosky, 1987 for theory and notation). This equation is equivalent to the Hasegawa-Mima equation describing drift-waves in an inhomogeneous plasma in a magnetic field (Hasegawa and Mima, 1977). Longuet-Higgins and Gill (1967) examined the interactions between planetary Rossby waves in the atmosphere and derived detailed conditions for three-wave resonance. The correspondence between Rossby waves in the atmosphere and drift waves in plasma have been thoroughly explored by Horton and Hasegawa (1994). Resonant wave-triad interactions play an essential role in the generation of turbulence and in determining the statistics of the power spectrum. Both energy and enstrophy are conserved in fluid systems governed by the Charney equation (2.20). 
Laser-matter interaction. Equations (2.11)-(2.13) are also equivalent to the Maxwell-Schrödinger envelope equations for the interaction between radiation and a two-level resonant medium in a microwave cavity. Holm and Kovačič (1992) show that perturbations of this system lead to homoclinic chaos, but we shall not explore that issue here. Wersinger et al. (1980) used a forced and damped version of the three-wave equations to study instability saturation by nonlinear mode coupling, and found irregular solutions indicating the presence of a strange attractor. See also Ott (1993) and Holm, Kovačič and Wettergren $(1995,1996)$ for more detailed studies of the perturbed three-wave system.

Nonlinear optics. The three-wave system also describes the dynamics of the envelopes of light-waves interacting quadratically in nonlinear material. The system has been examined in a series of recent papers (Alber, et al., 1998a,b; Luther et al., 2000) using a geometrical approach which allowed the reduced dynamics for the wave intensities to be represented as motion on a closed surface in three dimensions - the three-wave surface. Information about the corresponding reconstruction phases was recovered using the theory of connections on principal bundles.

In the special case $H=0$ the system (2.11)-(2.13) reduces to three real equations. Let

$$
A=i X_{1} \exp \left(i \phi_{1}\right), \quad B=i X_{2} \exp \left(i \phi_{2}\right), \quad C=i X_{3} \exp \left(i\left(\phi_{1}+\phi_{2}\right)\right)
$$

where $X_{1}, X_{2}$ and $X_{3}$ are real and the phases $\phi_{1}$ and $\phi_{2}$ are constants. The modulation equations become

$$
\dot{X}_{1}=-X_{2} X_{3}, \quad \dot{X}_{2}=-X_{3} X_{1}, \quad \dot{X}_{3}=+X_{1} X_{2} .
$$

We note that these equations are re-scaled versions of the Euler equations for the rotation of a free rigid body. The dynamics in this special case is expressible as motion on $\mathbf{R}^{3}$ namely,

$$
\dot{\mathbf{X}}=\frac{1}{8} \nabla J \times \nabla N=\frac{1}{4} \nabla N_{+} \times \nabla N_{-} .
$$

Considering the constancy of $J$ and $N$, we can describe a trajectory of the motion as an intersection between a hyperbolic cylinder $(J$ constant, see (2.19)) and an oblate spheroid ( $N$ constant; see (2.18) ). Eqn. (2.22) provides an alternative description. Here we have used the freedom in the $\mathbf{R}^{3}$ Poisson bracket exploited by David and Holm (1992) to represent the equations of motion on the intersection of two orthogonal circular cylinders, the level surfaces of the Manley-Rowe quantities, $N_{+}$and $N_{-}$. The invariance of the trajectories means that while the level surfaces of $J$ and $N$ differ from those of $N_{+}$and $N_{-}$, their intersections are precisely the same. For this particular value of $H=0$, the motion may be further reduced by expressing it in the coordinates lying on one of these two cylinders. See Holm and Marsden (1991) for the corresponding transformation of rigid body motion into pendular motion. See David and Holm (1992), and Alber et al. (1998a,b) for discussions of geometric phases in this situation. 


\subsection{Reduction of the system \& Reconstruction of the solution}

To reduce the system for $H \neq 0$, we employ a further canonical transformation, introduced in Holm and Kovačič, 1992. Namely, we set

$$
\begin{aligned}
& A=|A| \exp (i \xi), \\
& B=|B| \exp (i \eta), \\
& C=Z \exp (i(\xi+\eta)) .
\end{aligned}
$$

This transformation is canonical - it preserves the symplectic form

$$
d A \wedge d A^{*}+d B \wedge d B^{*}+d C \wedge d C^{*}=d Z \wedge d Z^{*} .
$$

In these variables, the Hamiltonian is a function of only $Z$ and $Z^{*}$

$$
H=\frac{1}{2}\left(Z+Z^{*}\right) \cdot \sqrt{N_{+}-|Z|^{2}} \cdot \sqrt{N_{-}-|Z|^{2}} .
$$

The Poisson bracket is $\left\{Z, Z^{*}\right\}=-2 i$ and the canonical equations reduce to

$$
i \dot{Z}=i\{Z, H\}=2 \frac{\partial H}{\partial Z^{*}} .
$$

This provides the slow dynamics of both the amplitude and phase of $Z=|Z| e^{i \zeta}$.

The amplitude $|Z|=|C|$ is obtained in closed form in terms of Jacobi elliptic functions as the solution of

$$
\left(\frac{d \mathcal{Q}}{d \tau}\right)^{2}=\left[\mathcal{Q}^{3}-2 \mathcal{Q}^{2}+\left(1-\mathcal{J}^{2}\right) \mathcal{Q}+2 \mathcal{E}\right],
$$

where $\mathcal{Q}=2|Z|^{2} / N, \mathcal{J}=J / N, \mathcal{E}=-4 H^{2} / N^{3}$ and $\tau=\sqrt{2 N} t$. This is equivalent to Eqn. (75) in Lynch, 2001 (an explicit expression for the solution in terms of elliptic functions is given in that paper). Once $|Z|$ is known, $|A|$ and $|B|$ follow immediately from the Manley-Rowe relations,

$$
|A|=\sqrt{N_{+}-|Z|^{2}}, \quad|B|=\sqrt{N_{-}-|Z|^{2}} .
$$

The phases $\xi$ and $\eta$ may now be determined. Using the three-wave equations (2.11)(2.13) together with (2.23)-(2.25), one finds

$$
\dot{\xi}=-\frac{H}{|A|^{2}}, \quad \dot{\eta}=-\frac{H}{|B|^{2}},
$$

so that $\xi$ and $\eta$ can be obtained by quadratures. Finally, the phase $\zeta$ of $Z$ is determined unambiguously by

$$
\frac{d|Z|^{2}}{d t}=-2 H \tan \zeta \quad \text { and } \quad H=|A||B||Z| \cos \zeta .
$$

Hence, we can now reconstruct the full solution as,

$$
A=|A| \exp (i \xi), \quad B=|B| \exp (i \eta), \quad C=|Z| \exp (i(\xi+\eta+\zeta)) .
$$




\section{Phase portraits.}

Consider the plane $\mathcal{C}$ in phase-space defined by $A=B=0$. This is a plane of unstable equilibrium points, representing purely vertical oscillations of the spring. The Hamiltonian vanishes identically on this plane, as does the angular momentum $J$. Each point $c_{0}$ in $\mathcal{C}$ has a heteroclinic orbit linking it to its antipodal point $-c_{0}$. Thus, the plane $\mathcal{C}$ of critical points is connected to itself by heteroclinic orbits. In Fig. 2, the horizontal plane is $\mathcal{C}$ and the vertical plane contains heteroclinic orbits from $c_{0}$ to $-c_{0}$. The vertical axis is $R=\sqrt{|A|^{2}+|B|^{2}}$. Since $N=R^{2}+2|C|^{2}$ is constant, each heteroclinic orbit is a semi-ellipse. Motion starting on one of these semi-ellipses will move towards an end-point, taking infinite time to reach it.

In Fig. 3 taken from Holm and Kovačič 1992, we present another view of the trajectories for $J=0$. The Hamiltonian is

$$
H=\frac{1}{2}\left(Z+Z^{*}\right) \cdot\left(\frac{1}{2} N-|Z|^{2}\right) .
$$

Accessible points lie on or within the circle $|Z|^{2}=N / 2$. For $H=0$ the trajectory is the segment of the imaginary axis within the circle. This is the homoclinic orbit. For $H \neq 0$, we solve for the imaginary part of $Z=Z_{1}+i Z_{2}$,

$$
Z_{2}= \pm \sqrt{-Z_{1}^{2}+\frac{1}{2} N-\left(H / Z_{1}\right)}
$$

This allows us to plot the trajectories for the range of $H$ for which real solutions exist. There are two equilibrium points, at $Z= \pm \sqrt{N / 6}$, corresponding to solutions for which there is no exchange of energy between the vertical and horizontal components. These are the cup-like and cap-like solutions first discussed by Vitt and Gorelik (1933).

\subsection{Geometry of the motion for fixed $J$}

The vertical amplitude is governed by equation (2.26), which we write as

$$
\frac{1}{2}\left(\frac{d \mathcal{Q}}{d \tau}\right)^{2}+\mathcal{V}(\mathcal{Q})=\mathcal{E},
$$

with the potential $\mathcal{V}(\mathcal{Q})$ given by

$$
\mathcal{V}(\mathcal{Q})=-\frac{1}{2}\left[\mathcal{Q}^{3}-2 \mathcal{Q}^{2}+\left(1-\mathcal{J}^{2}\right) \mathcal{Q}\right] .
$$

We note that $\mathcal{V}(\mathcal{Q})$ has three zeros, $\mathcal{Q}=0, \mathcal{Q}=1-\mathcal{J}$ and $\mathcal{Q}=1+\mathcal{J}$. Eqn. (3.1) is an energy equation for a particle of unit mass, with position $\mathcal{Q}$ and energy $\mathcal{E}$, moving in a cubic potential field $\mathcal{V}(\mathcal{Q})$. In Fig. 4 we plot $\mathcal{Q}$, given by (3.1), against $\mathcal{Q}$ for the cases $\mathcal{J}=0$ (left panel) and $\mathcal{J}=0.25$ (right panel), for a range of values of $\mathcal{E}$. Each curve represents the projection onto the reduced phase-space of the trajectory of the modulation envelope. The centers are relative equilibria, corresponding to the elliptic-parabolic solutions of (Lynch, 2001), which are generalizations of the cuplike and cap-like solutions of Vitt and Gorelik (1933). The case $\mathcal{J}=0$ includes the homoclinic trajectory, for which $H=0$. 


\subsection{Geometry of the motion for $H=0$}

For arbitrary $\mathcal{J}$, the $H=0$ motions are on a surface in the space with coordinates $(\mathcal{Q}, \dot{\mathcal{Q}}, \mathcal{J})$. This surface is depicted in Fig. 5. It has three singular points (i.e., it is equivalent to a sphere with three pinches) and its shape is similar to a tricorn hat. The motion takes place on an intersections of this surface with a plane of constant $\mathcal{J}$. There are three equilibrium solutions: that with $\mathcal{J}=0$ (marked H.P. in Fig. 5) is at the extremity of the homoclinic trajectory, and corresponds to purely vertical oscillatory motion; those with $\mathcal{J}= \pm 1$ correspond to purely horizontal motion, clockwise or anti-clockwise, with the spring tracing out a cone. The purely vertical motion is unstable; the conical motions are stable (perturbations about conical motion were investigated by Lynch, 2001). The dynamics on the tricorn are similar to the motion of a free rigid body. The three singular points correspond to the steady states of rotation about the three principal axes.

\subsection{Three-wave surfaces}

There is another way to depict the motion in reduced phase-space. Let us consider a reduced phase-space with $x$ and $y$ axes $X=\Re\left\{A B C^{*}\right\}$ and $Y=\Im\left\{A B C^{*}\right\}$ and $z$-axis $\mathcal{Q}=2|Z|^{2} / N$. We note that $X \equiv H$. It follows from $(2.17)-(2.19)$ that

$$
X^{2}+Y^{2}=|A|^{2}|B|^{2}|C|^{2}=\frac{1}{4}|Z|^{2}\left[\left(2|Z|^{2}-N\right)^{2}-J^{2}\right] .
$$

We define $\mathcal{X}=\left(2 / N^{3 / 2}\right) X$ and $\mathcal{Y}=\left(2 / N^{3 / 2}\right) Y$ and can write

$$
\mathcal{X}^{2}+\mathcal{Y}^{2}=\frac{1}{2}\left[\mathcal{Q}^{3}-2 \mathcal{Q}^{2}+\left(1-\mathcal{J}^{2}\right) \mathcal{Q}\right]=-\mathcal{V}(\mathcal{Q})
$$

where $\mathcal{V}$ is defined in (3.2). We note that $\mathcal{X}^{2}=-\mathcal{E}$ and $\mathcal{Y}^{2}=\frac{1}{2}(d \mathcal{Q} / d \tau)^{2}$. Eqn. (3.3) implies that the motion takes place on a surface of revolution about the $\mathcal{Q}$-axis. The radius for a given value of $\mathcal{Q}$ is the square-root of the cubic $-\mathcal{V}(\mathcal{Q})$. The physically assessable region is $0 \leq \mathcal{Q} \leq 1-|\mathcal{J}|$. Several such surfaces (for $\mathcal{J} \in\{0.0,0.1,0.2,0.3\}$ ) are shown in Fig. 6. Since $\mathcal{X}^{2}=\mathcal{H}^{2}=4 H^{2} / N^{3}$, the motion for given $\mathcal{J}$ takes place on the intersection of the corresponding surface of revolution with a plane of constant $\mathcal{X}$.

We can relate the tricorn surface to the surface of revolution. The former is appropriate for $H=0$; the $H \neq 0$ case is represented by trajectories inside this surface. If we slice the tricorn surface in a plane of fixed $\mathcal{J}$ we get a set of closed trajectories, the outside one for $H=0$ the others for $H \neq 0$ (the cases $\mathcal{J}=0$ and $\mathcal{J}=0.25$ are plotted in Fig. 4 above.) If we now distort the $\mathcal{J}$-section into a cup-like surface, by taking $H$ as a vertical coordinate and plotting each trajectory at a height depending on its $H$ value, we get half of a closed surface. Each trajectory is selected by a $H$-plane section. Alteration of the sign of $H$ corresponds to reversal of time. Completing the surface by reflection in the plane $H=0$ gives the surface generated

by rotating the root-cubic graph $\sqrt{-\mathcal{V}(\mathcal{Q})}$ about the $\mathcal{Q}$-axis, i.e., the surface given by (3.3). These surfaces are what Alber, et al., (1998a) call the three-wave surfaces. They foliate the volume contained within the surface for $\mathcal{J}=0$. 


\section{The Precession of the swing plane}

The characteristic feature of the behavior of the physical spring is its stepwise precession, which we shall now analyze. As the oscillations change from horizontal to vertical and back again, it is observed that each successive horizontal excursion departs in a different direction. The only reference to this phenomenon of which we are aware, prior to Lynch (2001), is Cayton (1977). Cayton briefly discussed three-dimensional solutions and mentioned the precession of the swing plane, but did not analyze its dynamics. Surprisingly, the characteristic stepwise precession of the swinging spring has been largely ignored, although it is immediately obvious upon observation of a physical elastic pendulum with $\omega_{Z} \approx 2 \omega_{R}$. Indeed, this precession is almost impossible to suppress experimentally when the initial motion is close to vertical.

\subsection{Qualitative description}

If the horizontal projection of the motion is an ellipse of high eccentricity, the motion is approximately planar. We call the vertical plane through the major axis of this ellipse the swing plane. When the initial oscillations are quasi-vertical, the motion gradually develops into an essentially horizontal swinging motion. This horizontal swinging does not persist, but soon passes again into nearly vertical springing oscillations similar to the initial motion. Subsequently, a horizontal swing again develops, but now in a different direction. The stepwise precession of this exchange between springing and swinging motion continues indefinitely in the absence of dissipation and is the characteristic experimental feature of the swinging spring. We shall seek an expression for the change in direction of the swing plane from one horizontal excursion to the next.

A full knowledge of the solutions of the three equations of motion would of course suffice to determine the swing plane at each moment in time. In Lynch (2001) the equations were expressed in rotating co-ordinates, and a particular solution for the slow rotation of the swing plane was posited as a function of the vertical amplitude $|C|$, by assuming a certain angular relation. Following this assumption, the angle of the swing-plane could be expressed as an integral involving elliptic functions.

\subsection{Pattern evocation in shape space}

We shall approach the precession problem using pattern evocation in shape space. Pattern evocation seeks a relative equilibrium (in shape space) in which a phase relationship between the variables (the shape) is preserved (Marsden, et al., 1995, 1996). We track the pattern by moving to a non-uniformly rotating frame in which the orientation of the shape is fixed. This is a generalization of the idea of tracking a satellite orbit by evoking constancy of the areal velocity required to conserve angular momentum.

Our particular geometric assumption is that the angle between the complex amplitudes $a$ and $b$ remains constant, in an appropriately rotating frame. Writing these 
amplitudes in vector form as $\mathbf{a}=(|a| \cos \alpha,|a| \sin \alpha, 0), \mathbf{b}=(|b| \cos \beta,|b| \sin \beta, 0)$ and taking $\mathbf{k}=(0,0,1)$ yields

$$
J=-\mathbf{k} \cdot \mathbf{a} \times \mathbf{b}=|a b| \sin (\alpha-\beta), \quad \mathbf{a} \cdot \mathbf{b}=|a b| \cos (\alpha-\beta) .
$$

Consequently, our geometric pattern evocation assumption that the phase difference $\alpha-\beta$ remains constant immediately implies that $|a b|$ is also constant. The conservation of angular momentum $J$ means that the area of the parallelogram formed by the vectors $\mathbf{a}$ and $\mathbf{b}$ is constant. The requirement of constant $\alpha-\beta$ imposes an additional geometric constraint on the possible shape of the orbits. For example, when $\alpha-\beta=\pi / 2(\bmod \pi)$, the orbits are elliptical.

\subsection{Modulation equations in rotating coordinates}

We shall transform to rotating co-ordinates and seek an expression for the (slow) rotation frequency $\Omega(t)$ that allows us to estimate the stepwise precession of the swinging spring by imposing the pattern evocation constraint that $\alpha-\beta$ remains constant.

In a rotating frame, the approximate Lagrangian (2.1) at cubic order in the coordinate displacements becomes, with $\mathbf{x}=(x, y, z)$,

$$
L=\frac{1}{2}|\dot{\mathbf{x}}+\Omega(t) \hat{\mathbf{z}} \times \mathbf{x}|^{2}-\frac{1}{2}\left(\omega_{R}^{2}\left(x^{2}+y^{2}\right)+\omega_{Z}^{2} z^{2}\right)+\frac{1}{2} \lambda\left(x^{2}+y^{2}\right) z .
$$

Now $x, y$ and $z$ are Cartesian coordinates centered at the point of equilibrium in the rotating frame, $\omega_{R}=\sqrt{g / \ell}$ is the frequency of linear pendular motion, $\omega_{Z}=\sqrt{k / m}$ is the frequency of its elastic oscillations and $\lambda=\ell_{0} \omega_{Z}^{2} / \ell^{2}$. The corresponding EulerLagrange equations of motion (2.2)-(2.4) may be written in rotating coordinates as

$$
\begin{aligned}
\ddot{x}-\dot{\Omega}(t) y-2 \Omega(t) \dot{y}+\left(\omega_{R}^{2}-\Omega^{2}(t)\right) x & =\lambda x z, \\
\ddot{y}+\dot{\Omega}(t) x+2 \Omega(t) \dot{x}+\left(\omega_{R}^{2}-\Omega^{2}(t)\right) y & =\lambda y z, \\
\ddot{z}+\omega_{Z}^{2} z & =\frac{1}{2} \lambda\left(x^{2}+y^{2}\right) .
\end{aligned}
$$

The vertical component of angular momentum is

$$
h=\hat{\mathbf{z}} \cdot \mathbf{x} \times(\dot{\mathbf{x}}+\Omega(t) \hat{\mathbf{z}} \times \mathbf{x})=(x \dot{y}-\dot{x} y)+\Omega(t)\left(x^{2}+y^{2}\right)
$$

and is a constant of the motion for these equations. However, upon Legendretransforming, one finds the time-dependent Hamiltonian satisfies

$$
\dot{H}=-\dot{\Omega}(t) h .
$$

Thus, perhaps not unexpectedly, exact energy conservation breaks down to the extent that the rotation frequency is nonuniform. 


\subsection{Averaged Lagrangian and modulation equations for slow rotation}

The modulation equations in axes rotating with angular velocity $\Omega(t)$ about the vertical are obtained in the resonant case $\omega_{Z}=2 \omega_{R}$ by applying the averaged Lagrangian technique (Whitham, 1974). Accordingly, the solution of (4.2)-(4.4) is assumed to be of the form

$$
\begin{aligned}
x & =\Re\left[a(t) \exp \left(i \omega_{R} t\right)\right], \\
y & =\Re\left[b(t) \exp \left(i \omega_{R} t\right)\right], \\
z & =\Re\left[c(t) \exp \left(2 i \omega_{R} t\right)\right] .
\end{aligned}
$$

(Note that subscript-zeroes are dropped for these modulation amplitudes in the rotating frame.) In these variables, the averaged Lagrangian corresponding to (4.1) may be written as

$$
\begin{gathered}
\langle L\rangle=\frac{1}{2} \omega_{R}\left[\Im\left\{\dot{a} a^{*}+\dot{b} b^{*}+2 \dot{c} c^{*}\right\}+\Re\left\{\left(a^{2}+b^{2}\right) c^{*}\right\}+2 \Omega \Im\left\{a b^{*}\right\}\right] \\
+\frac{1}{2} \Omega \Re\left[a^{*} \dot{b}-\dot{a}^{*} b\right]+\frac{1}{4} \Omega^{2}\left[|a|^{2}+|b|^{2}\right] .
\end{gathered}
$$

On assuming that the rotation frequency is sufficiently slow that $\Omega / \omega_{R} \ll 1$, we shall neglect all terms in the averaged Lagrangian (4.8) that are not multiplied by $\omega_{R}$. In this approximation of slow rotation, the averaged Lagrangian is given by the simpler expression,

$$
\langle L\rangle=\frac{1}{2} \omega_{R}\left[\Im\left\{\dot{a} a^{*}+\dot{b} b^{*}+2 \dot{c} c^{*}\right\}+\Re\left\{\left(a^{2}+b^{2}\right) c^{*}\right\}+2 \Omega J\right] .
$$

Here $J=\Im\left\{a b^{*}\right\}$ is the angular momentum, a conserved quantity at this level of approximation and formally identical to the expression in non-rotating coordinates. The Euler-Lagrange modulation equations in this approximation may be written as

$$
\begin{aligned}
i \dot{a} & =a^{*} c+i \Omega b, \\
i \dot{b} & =b^{*} c-i \Omega a, \\
i \dot{c} & =\frac{1}{4}\left(a^{2}+b^{2}\right) .
\end{aligned}
$$

We may also write these leading order equations in Hamiltonian form. When $\langle H\rangle$ is defined by

$$
\langle H\rangle=\Re\left\{\left(a^{2}+b^{2}\right) c^{*}\right\}+2 \Omega \Im\left\{a b^{*}\right\},
$$

with coordinates $(a, b, c)$, conjugate momenta $\left(a^{*}, b^{*}, 2 c^{*}\right)$ and Poisson brackets defined by $\left\{a, a^{*}\right\}=\left\{b, b^{*}\right\}=2\left\{c, c^{*}\right\}=-i$, the modulation equations (4.10)-(4.12) are expressible in canonical Hamiltonian form as,

$$
i \dot{a}=i\{a,\langle H\rangle\}=\frac{\partial\langle H\rangle}{\partial a^{*}}, \quad i \dot{b}=i\{b,\langle H\rangle\}=\frac{\partial\langle H\rangle}{\partial b^{*}}, \quad i \dot{c}=i\{c,\langle H\rangle\}=\frac{\partial\langle H\rangle}{\partial 2 c^{*}} .
$$


The three constants of the motion for these equations are

$$
\begin{aligned}
H_{0} & =\frac{1}{2}\left[\left(a^{2}+b^{2}\right) c^{*}+\left(a^{* 2}+b^{* 2}\right) c\right]=\Re\left\{\left(a^{2}+b^{2}\right) c^{*}\right\}, \\
N & =|a|^{2}+|b|^{2}+4|c|^{2}, \\
J & =\left(a b^{*}-a^{*} b\right) / 2 i=\Im\left\{a b^{*}\right\} .
\end{aligned}
$$

We now introduce the pattern evocation assumption. Noting that

$$
|a b|^{2}=\left(\Re\left\{a b^{*}\right\}\right)^{2}+\left(\Im\left\{a b^{*}\right\}\right)^{2},
$$

and that the second term is just $J^{2}$, implies constancy of $\Re\left\{a b^{*}\right\}$. Using Eqns. (4.10) and (4.11) it follows that

$$
\frac{d}{d t}|a b|^{2}=-2 \Re\left\{a b^{*}\right\}\left[2 \Im\left\{a b c^{*}\right\}+\Omega\left(|a|^{2}-|b|^{2}\right)\right]=0 .
$$

Either factor may vanish, so there appear to be two possibilities for the solution. We first assume that the factor in square brackets in (4.16) vanishes. This implies

$$
\Omega=-\frac{2 \Im\left\{a b c^{*}\right\}}{|a|^{2}-|b|^{2}}=-\frac{2|a b c| \sin (\alpha+\beta-\gamma)}{|a|^{2}-|b|^{2}} .
$$

(where $c=|c| e^{i \gamma}$ ). The precession angle $\Theta=\int_{0}^{t} \Omega\left(t^{\prime}\right) d t^{\prime}$ can be ascertained by integrating $\Omega$ over the time interval of the motion. In the special case $\alpha-\beta=$ $\frac{\pi}{2}(\bmod \pi)$, one finds by using the constants of motion that

$$
\Omega=\frac{2 J H_{0}}{\left(N-4|c|^{2}\right)^{2}-4 J^{2}} .
$$

This case also corresponds to the vanishing of the first factor in (4.16), so that $\Re\left\{a b^{*}\right\}=0$ and $\mathbf{a}$ and $\mathbf{b}$ are $90^{\circ}$ out of phase. This was the Ansatz introduced by Lynch (2001). He showed that, in this case, the rotation rate is given by (4.18). We now see that the result in Lynch (2001) is a special case of the general result (4.17). In this special case, $\Omega$ can be computed as soon as $|c|$ is known. We will examine this case numerically below.

\subsection{The instantaneous ellipse}

In order to define precisely the precession angle, we introduce an ellipse which approximates the horizontal projection of the trajectory of the pendulum. Recall that the full solution for the horizontal components is

$$
x=\Re\left\{a \exp \left(i \omega_{R} t\right)\right\}=|a| \cos \left(\omega_{R} t+\alpha\right), \quad y=\Re\left\{b \exp \left(i \omega_{R} t\right)\right\}=|b| \cos \left(\omega_{R} t+\beta\right),
$$

where $\alpha$ and $\beta$ are the phases of $a$ and $b$. The amplitudes and phases are assumed to vary slowly. If they are regarded as constant over a period $\tau=1 / \omega_{R}$ of the fast motion, these equations describe a central ellipse,

$$
P x^{2}+2 Q x y+R y^{2}=S
$$


where $P=|b|^{2}, Q=-|a b| \cos (\alpha-\beta), R=|a|^{2}$ and $S=J^{2}$. The area of the ellipse is easily calculated and is found to have the constant value $\pi J$. Its orientation is determined by eliminating the cross-term in (4.19). This is achieved as usual by rotating the axes through an angle $\theta$, given by

$$
\tan 2 \theta=\frac{2 Q}{P-R}=\frac{2|a b| \cos (\alpha-\beta)}{|a|^{2}-|b|^{2}} .
$$

The semi-axes of the ellipse are given by

$$
A_{1}=\frac{J}{\sqrt{P \cos ^{2} \theta+Q \sin 2 \theta+R \sin ^{2} \theta}}, \quad A_{2}=\frac{J}{\sqrt{P \sin ^{2} \theta-Q \sin 2 \theta+R \cos ^{2} \theta}} .
$$

The area is $\pi A_{1} A_{2}=\pi J$ and the eccentricity can be calculated immediately. In the case of unmodulated motion, such as the elliptic-parabolic modes described in Lynch (2001), the instantaneous ellipse corresponds to the trajectory, which is a precessing ellipse. In general, it is only an approximation to the trajectory, but we may define the orientation or azimuth at any time to be the angle $\theta$ given by (4.20). This angle will be compared to the precession angle $\Theta$ calculated by integrating (4.18) and shown to give almost identical results.

\section{$5 \quad$ Numerical results}

We examine the results of numerical integrations of the modulation equations (2.8)(2.10) and compare them to the solutions of the exact equations (2.2)-(2.4). It will be seen that the modulation equations provide an excellent description of the envelope of the rapidly varying solution of the full equations. We then compare the stepwise precession angle predicted by a formula based on constancy of the angle $\alpha-\beta$ with the numerical simulation of this quantity and show that the two values track each other essentially exactly.

The parameter values chosen for all numerical integrations are $m=1 \mathrm{~kg}, \ell=1 \mathrm{~m}$, $g=\pi^{2} \mathrm{~m} \mathrm{~s}^{-2}$ and $k=4 \pi^{2} \mathrm{~kg} \mathrm{~s}^{-2}$ so that $\omega_{R}=\pi, \omega_{Z}=2 \pi$ and the resonance condition $\omega_{Z}=2 \omega_{R}$ holds. The linear rotational mode has period $\tau_{R}=2 \mathrm{~s}$, and the vertical mode has period $\tau_{Z}=1 \mathrm{~s}$. The initial conditions are set as follows,

$$
\left(x_{0}, y_{0}, z_{0}\right)=(0.006,0,0.012) ; \quad\left(\dot{x}_{0}, \dot{y}_{0}, \dot{z}_{0}\right)=(0,0.00489,0) .
$$

(The value of $\dot{y}_{0}$ was chosen to tune the precession angle to be an even fraction of $180^{\circ}$, making the amplitudes, though not the phases, periodic). The corresponding initial values for the modulation equations (2.8)-(2.10) are given by

$$
\begin{gathered}
\alpha_{0}=\arctan \left(\frac{-\dot{x}_{0}}{\omega_{R} x_{0}}\right), \quad \beta_{0}=\arctan \left(\frac{-\dot{y}_{0}}{\omega_{R} y_{0}}\right), \quad \gamma_{0}=\arctan \left(\frac{-\dot{z}_{0}}{2 \omega_{R} z_{0}}\right), \\
\left|a_{0}\right|=\left(\frac{x_{0}}{\cos \alpha_{0}}\right), \quad\left|b_{0}\right|=-\left(\frac{\dot{y}_{0}}{\omega_{R} \sin \beta_{0}}\right), \quad\left|c_{0}\right|=\left(\frac{z_{0}}{\cos \gamma_{0}}\right),
\end{gathered}
$$


giving the values $\left(\left|a_{0}\right|,\left|b_{0}\right|,\left|c_{0}\right|\right)=(0.006,0.002,0.012)$ and $\left(\alpha_{0}, \beta_{0}, \gamma_{0}\right)=(0,-\pi / 2,0)$. The constants of the motion take the following values,

$$
H=4.03 \times 10^{-7}, \quad J=9.34 \times 10^{-6}, \quad N=6.14 \times 10^{-4} .
$$

The integration was extended over a period of 1000 seconds (i.e., 1000 vertical oscillations). As a check on numerical accuracy, the changes in these quantities, which should remain constant, was calculated, with the following results:

$$
\left(\frac{H_{\text {Final }}}{H_{\text {Initial }}}\right)=100.04 \%, \quad\left(\frac{J_{\text {Final }}}{J_{\text {Initial }}}\right)=99.997 \%, \quad\left(\frac{N_{\text {Final }}}{N_{\text {Initial }}}\right)=100.00 \% \text {. }
$$

We now directly compare the solutions of the 'exact' equations (2.2)-(2.4) and the 'approximate' or modulation equations (2.8)-(2.10). Once the modulation equations have been solved for the envelope amplitudes and phases, the full approximate solution is given by (2.5) $-(2.7)$. We first consider the horizontal projection of the solution for the 1000 second integration. This is the period required for the solution to precess through approximately $180^{\circ}$. In Fig. 7 (top panel) we plot $x$ versus $y$ for the exact solution. In Fig. 7 (bottom panel) we plot the corresponding solution from the modulation equations. It is clear that there is great similarity between the two solutions; indeed, the two plots are indistinguishable. The precession angle between horizontal excursions is close to $30^{\circ}$ (the value of $\dot{y}_{0}$ was chosen to ensure this). The modulation period is approximately 167 seconds; thus, the instantaneous ellipse rotates through six cycles and $180^{\circ}$ in 1000 seconds.

The vertical structure of the solution is displayed in Fig. 8, where $z$ for the exact solution (top panel) and $\Re\left\{c_{0}(t) \exp \left(2 i \omega_{R} t\right)\right\}$ for the approximate solution (bottom panel) are seen to be virtually identical. For clarity, the solutions are plotted only for the first modulation cycle of 167 seconds. The character of the solution - rapid oscillations with a slowly-varying amplitude envelope - is clear from the figure. The vertical amplitude is close to zero when horizontal excursions are at their peak. This is confirmed in Fig. 9 (top panel) where the horizontal modulation amplitude $S=\sqrt{|a|^{2}+|b|^{2}}$ and vertical modulation amplitude $C=|c|$ are plotted against time.

In Fig. 9 (bottom panel) we plot the squared eccentricity $e^{2}=\left(1-A_{\text {min }}^{2} / A_{\text {maj }}^{2}\right)$ of the envelope of the horizontal projection of the approximate solution, where the semi-axes $A_{\text {maj }}$ and $A_{\text {min }}$ are calculated from (4.21). The eccentricity is close to unity for most of the integration. Horizontal excursions of the pendulum occur during this time. For short periods, when the horizontal amplitude is minimum, the value of $e$ drops significantly (solid line). During this time, the angular velocity, calculated as the rate of change of the azimuth given by (4.20), reaches a maximum (dashed line). Thus, the precession occurs in bursts near the times when the vertical amplitude is maximum and horizontal amplitude minimum.

The stepwise nature of the precession is clearly illustrated in Fig. 10. The azimuthal angle $\vartheta$ of the numerical solution of the exact equations may be calculated by fitting a central conic to every three consecutive points on the trajectory. Assuming a solution of the form

$$
\tilde{P} x^{2}+2 \tilde{Q} x y+\tilde{R} y^{2}=1
$$


and requiring that the three points lie on this curve, we obtain three equations for the coefficients $(\tilde{P}, \tilde{Q}, \tilde{R})$. ¿From these, the azimuth $\vartheta$ and the semi-axes are obtained from equations analogous to (4.20) and (4.21). This is compared in Fig. 10 to the corresponding value $\theta$ resulting from integration of the modulation equations. It is noteworthy that $\vartheta$ and $\theta$ remain quasi-constant for most of the modulation cycle, changing rapidly only over short intervals around the times when $C$ is maximum and $S$ is minimum. The advances in phase are very similar for the exact and approximate solutions. However, there are small differences: $\theta-\vartheta$ is also plotted in Fig. 10 (dotted line). This sensitive quantity reaches its maximum value of $4.35^{\circ}$ at the end of the integration.

The azimuthal change between successive horizontal excursions is very close to $30^{\circ}$ for both exact and approximate solutions. We also calculated the angle $\Theta$ resulting from an integration of (4.18). The graphs of $\theta$ and $\Theta$ (not plotted) are indistinguishable. The maximum difference $|\theta-\Theta|$ was only $0.0063^{\circ}$. This is remarkable: the value $\Theta$ derived from (4.18) involves an assumption that $\alpha-\beta$ is constant in a particular rotating frame. The azimuth $\theta$ from the modulation equations makes no such assumption, yet the two solutions are practically identical. This confirms that the pattern evocation assumption which yields the result (4.18) is sound.

Numerous other integrations of the exact and modulation equations were also carried out. They confirm that the stepwise precession of the azimuthal angle is a distinct characteristic of the swinging spring. This is also in complete agreement with simple experiments with a physical pendulum, where the periodic exchange of energy between horizontal and vertical and the precession of the swing plane between horizontal excursions are the main observable properties of the motion.

\section{Acknowledgements}

The authors would like to acknowledge the facilities provided by the Isaac Newton Institute at Cambridge University, where this work began. Peter Lynch would like to thank the Center for Nonlinear Studies, Los Alamos National Laboratory, for support and hospitality. Darryl Holm is grateful to Gregor Kovačič and Jerry Marsden for many fruitful discussions and ongoing collaborations in dynamical systems analysis. DDH was supported by US DOE, under contract W-7405-ENG-36.

\section{References}

Aceves, A B, D D Holm, G Kovačič and I Timofeyev, 1997: Homoclinic orbits and chaos in a second-harmonic generating optical cavity. Phys. Lett. A 233, 203-208.

Alber, M S, G G Luther, J E Marsden and J M Robbins, 1998a: Geometric phases, reduction and Lie-Poisson structure for the resonant three-wave interaction, Physica D 123, 271-290.

Alber, M S, G G Luther, J E Marsden and J M Robbins, 1998b: Geometry and Control of ThreeWave Interactions. (Caltech preprint) 
Bretherton, F P, 1964: Resonant interactions between waves. The case of discrete oscillations. J Fluid Mech., 20, 457-479.

Cayton, Th E, The laboratory spring-mass oscillator: an example of parametric instability. Am. J. Phys., 45, 723-732 (1977).

David, D and D D Holm: Multiple Lie-Poisson Structures, Reductions, and Geometric Phases for the Maxwell-Bloch Traveling-Wave Equations, J. Nonlin. Sci. 2 (1992) 241-262.

David, D, D D Holm and M Tratnick: Hamiltonian Chaos in Nonlinear Optical Polarization Dynamics, Phys. Rep. 187 (1990) 281-367.

Hasegawa, A and K Mima, 1977: Pseudo-three-dimensional turbulence in magnetized nonuniform plasmas. Phys. Fluids, 21(1), 87-92.

Holm, D D and G Kovačič, 1992: Homoclinic chaos in a laser-matter system. Physica D 56, 270-300.

Holm, D D, G Kovačič and T A Wettergren, 1995: Near integrability and chaos in a resonant-cavity laser model. Phys. Lett. A, 200, 299-307.

Holm, D D, G Kovačič and T A Wettergren, 1996: Homoclinic orbits in the Maxwell-Bloch equations with a probe. Phys. Rev. E, 54, 243-256.

Holm, D D and J E Marsden, 1991: The rotor and the pendulum. in Symplectic Geometry and Mathematical Physics, P. Donato, C. Duval, J. Elhadad, G. M. Tuynman, ed., Prog. in Math. Vol. 99, Birkhauser: Boston, 1991, pp. 189-203.

Horton, W and A Hasegawa, 1994: Quasi-two-dimensional dynamics of plasmas and fluids. Chaos, $4(2), 227-251$.

Longuet-Higgins, M S and A E Gill, 1967: Resonant interactions between planetary waves Proc. Roy. Soc., A299, 120-140.

Lorenz, E N, 1963: Deterministic non-periodic flow. J. Atmos. Sci., 20, 130-141.

Luther, G G, M S Alber, J E Marsden and J M Robbins, 2000: Geometric analysis of optical frequency conversion and its control in quadratic nonlinear media. J. Optical Soc. Am., B/17, 932-941.

Lynch, P, 2001: Resonant motions of the three-dimensional elastic pendulum. To appear in Intl. J. Nonlin. Mech. (2001).

Marsden, J E, J Scheurle and J M Wendlandt, 1996: Visualization of orbits and pattern evocation for the double spherical pendulum. ICIAM 95 (Hamburg, 1995), 213-232, Math. Res., 87, Akademie Verlag, Berlin, 1996.

Marsden, J E and J Scheurle, 1995: Pattern evocation and geometric phases in mechanical systems with symmetry. Dynam. Stability Systems, 10(4), 315-338.

Montgomery, R, 1991: How much does the rigid body rotate? A Berry's phase from the 18th century. Am. J. Phys., 59(5), 394-398.

Ott, Edward, 1993: Chaos in Dynamical Systems. Cambridge Univ. Press, 385pp.

Pedlosky, J, 1987: Geophysical Fluid Dynamics. Springer-Verlag, 710pp.

Sparrow, C., 1982: The Lorenz Equations: Bifurcations, Chaos and Strange Attractors. SpringerVerlag, New York, 269pp.

Vitt, A and G Gorelik, Kolebaniya uprugogo mayatnika kak primer kolebaniy dvukh parametricheski svyazannykh linejnykh sistem. Zh. Tekh. Fiz. (J. Tech. Phys.) 3(2-3), 294-307 (1933). Available in English translation: Oscillations of an Elastic Pendulum as an Example of the Oscillations of Two Parametrically Coupled Linear Systems. Translated by Lisa Shields, with an Introduction by Peter Lynch. Historical Note No. 3, Met Éireann, Dublin (1999).

Wersinger, J-M, J M Finn and E Ott, 1980: Bifurcations and strange behavior in instability saturation by nonlinear mode coupling. Phys. Rev. Lett., 44, 453-456.

Whitham, G N, 1974: Linear and Nonlinear Waves, John Wiley \& Sons. 


\section{Figure Captions}

Figure 1. Schematic diagram of the elastic pendulum or swinging spring. Cartesian coordinates centered at the position of equilibrium are used.

Figure 2. $\mathcal{C}$ is the plane of critical points, $A=0=B$. The vertical axis is $R=$ $\sqrt{|A|^{2}+|B|^{2}}$. The vertical plane contains heteroclinic semi-ellipses passing from $c_{0}$ to $-c_{0}$.

Figure 3. Phase portrait in the $Z$-plane for $J=0$. The motion is confined within the circle $|Z|^{2}=\frac{1}{2} N$. The segment of the imaginary axis within this circle is the homoclinic orbit.

Figure 4. Plots of $\dot{\mathcal{Q}}$ versus $\mathcal{Q}$ for $\mathcal{J}=0$ and $\mathcal{J}=0.25$, for a range of values $E \in\{-0.0635,-0.0529,-0.0423,-0.0317,-0.0212,-0.0106,0\}$.

Figure 5. Tricorn surface, upon which motion takes place when $H=0$. The coordinates are $\mathcal{J}, \mathcal{Q}, \dot{\mathcal{Q}}$. The motion takes place on the intersections of this surface with a plane of constant $\mathcal{J}$ (such planes are indicated by the stripes). This surface has three singular points. The homoclinic point is marked H.P.

Figure 6. Surfaces of revolution about the $\mathcal{Q}$-axis, for $\mathcal{J} \in\{0.0,0.1,0.2,0.3\}$. The radius for given $\mathcal{Q}$ is given by the square-root of the cubic $-\mathcal{V}(\mathcal{Q})$. For given $\mathcal{J}$, the motion takes place on the intersection of the corresponding surface with a plane of constant $X$.

Figure 7. Horizontal projection of the solution for an integration of 1000 seconds. Top: solution of the 'exact' equations. Bottom: solution of the 'approximate' equations.

Figure 8. Vertical amplitude of the solution for the first modulation cycle (first 167 seconds). Top: solution of the 'exact' equations. Bottom: solution of the 'approximate' equations.

Figure 9. Top panel: Envelope amplitude of the approximate solution. $S=$ $\sqrt{|a|^{2}+|b|^{2}}$ (solid line) and $C=|c|$ (dashed line). Bottom panel: Square of the eccentricity (solid line) and angular velocity $\Omega$ (scaled by 50) of the instantaneous ellipse (dashed line).

Figure 10. Azimuth angle (in degrees) for the 'exact' solution ( $\vartheta$, solid line) and the 'approximate' solution ( $\theta$, dashed line). The difference $\theta-\vartheta$ is plotted as a dotted line. The azimuth $\Theta$ resulting from integration of (4.18) (not plotted) is indistinguishable from the values $\theta$ of the approximate solution. 


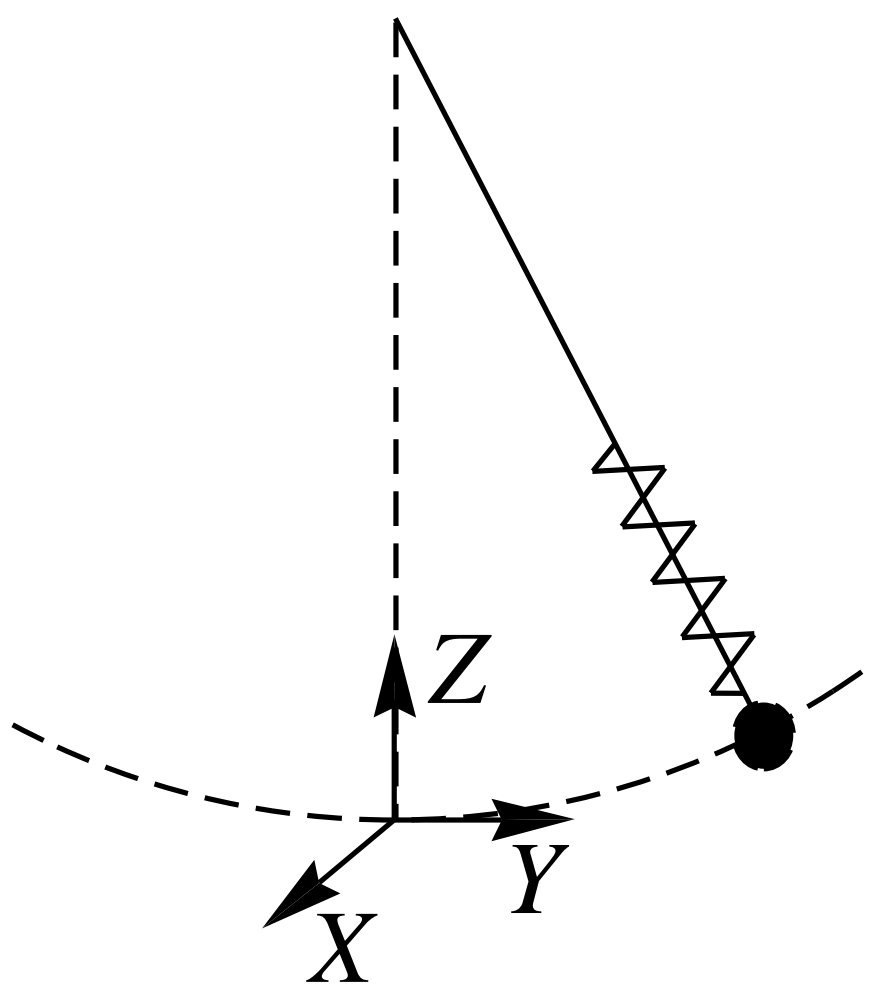

Figure 1. Schematic diagram of the elastic pendulum, or swinging spring. Cartesian coordinates centered at the position of equilibrium are used. 


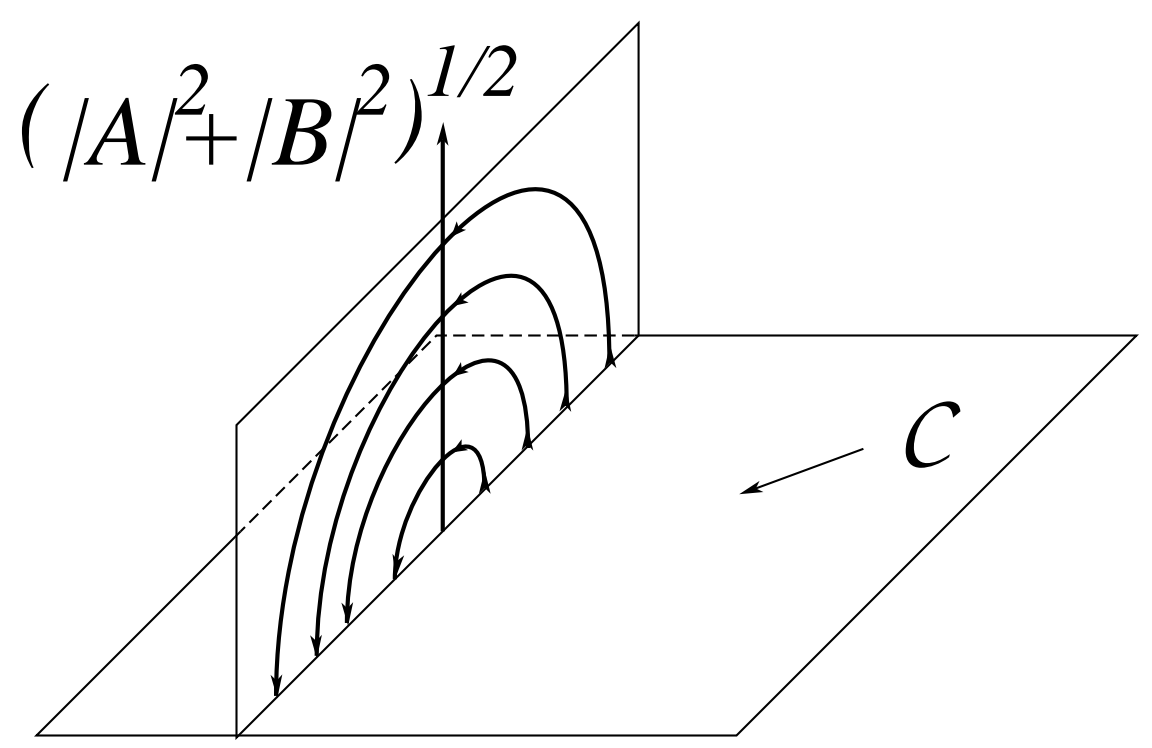

Figure 2. $\mathcal{C}$ is the plane of critical points, $A=0=B$. The vertical axis is $R=$ $\sqrt{|A|^{2}+|B|^{2}}$. The vertical plane contains heteroclinic semi-ellipses passing from $c_{0}$ to $-c_{0}$. 


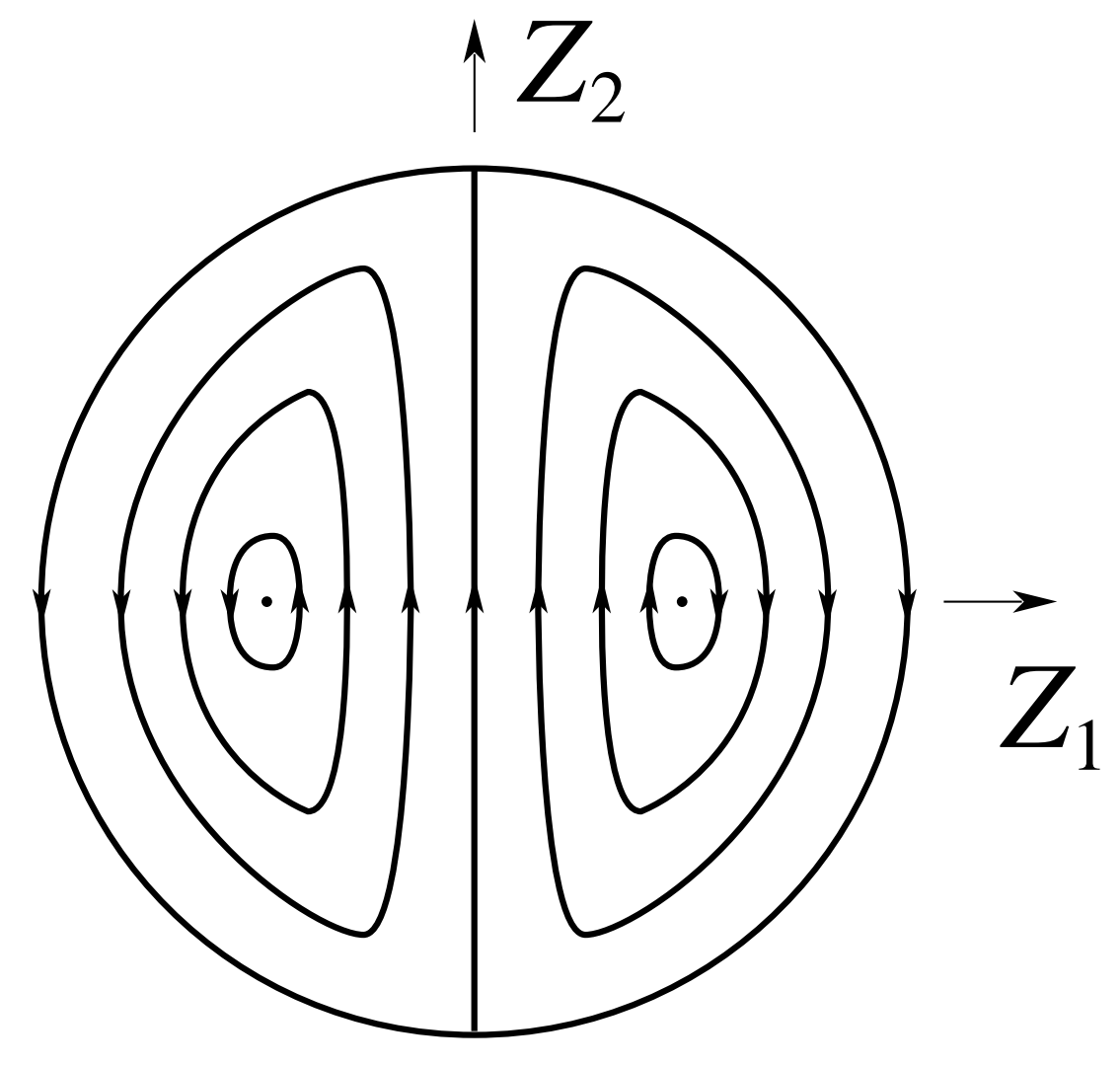

Figure 3. Phase portrait in the $Z$-plane for $J=0$. The motion is confined within the circle $|Z|^{2}=\frac{1}{2} N$. The segment of the imaginary axis within this circle is the homoclinic orbit. 

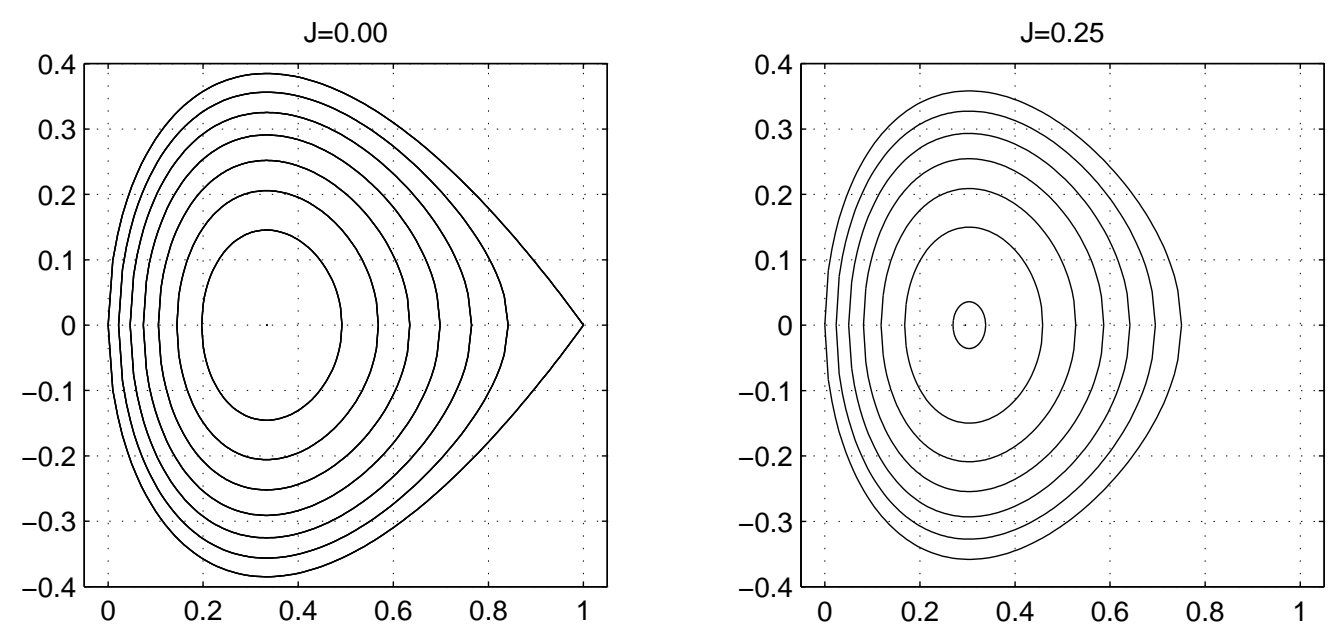

Figure 4. Plots of $\dot{\mathcal{Q}}$ versus $\mathcal{Q}$ for $\mathcal{J}=0$ and $\mathcal{J}=0.25$, for a range of values $E \in\{-0.0635,-0.0529,-0.0423,-0.0317,-0.0212,-0.0106,0\}$. 


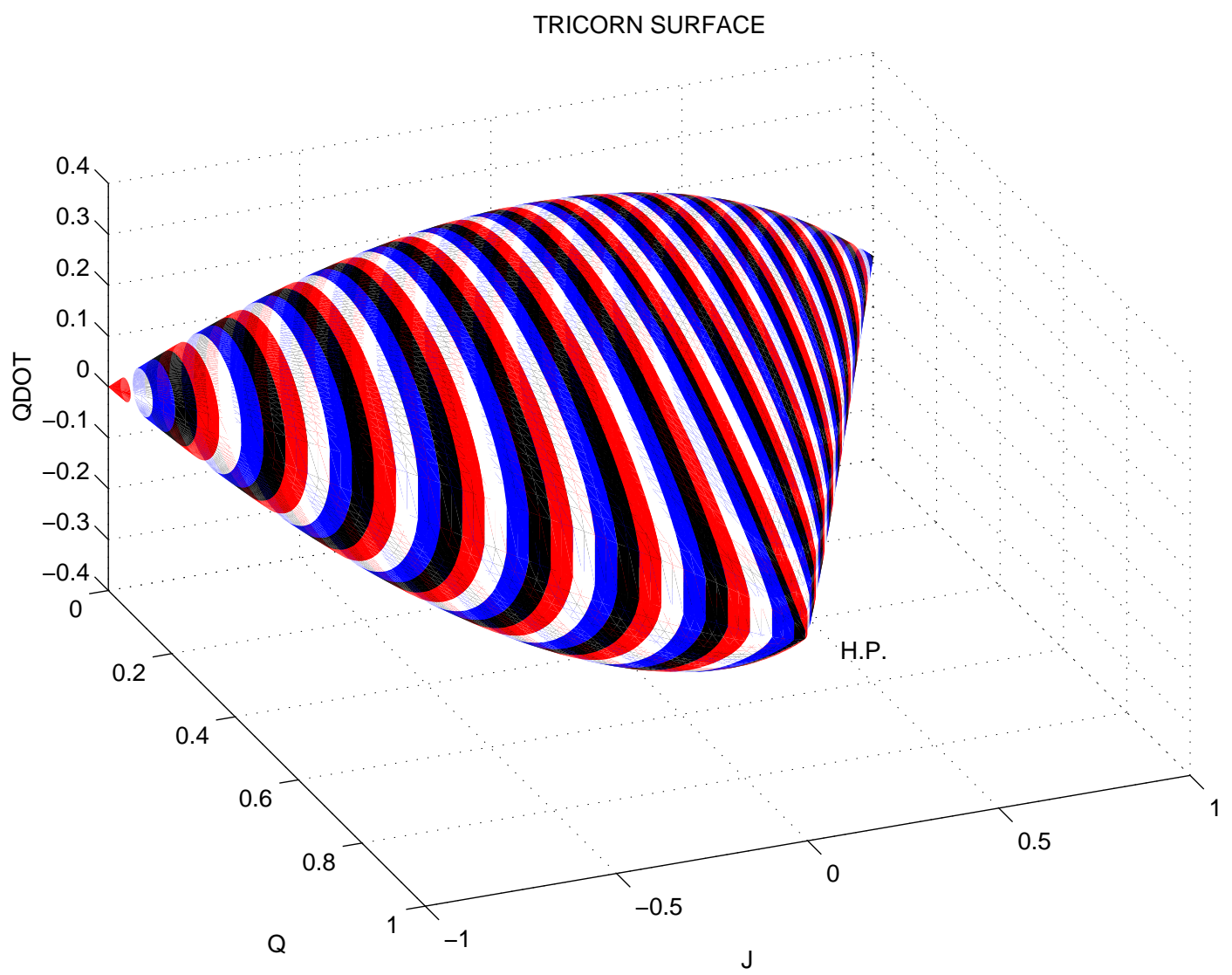

Figure 5. Tricorn surface, upon which motion takes place when $H=0$. The coordinates are $\mathcal{J}, \mathcal{Q}, \dot{\mathcal{Q}}$. The motion takes place on the intersections of this surface with a plane of constant $\mathcal{J}$ (such planes are indicated by the stripes). This surface has three singular points. The homoclinic point is marked H.P. 
THREE-WAVE SURFACE, $\mathrm{J}=0.0$

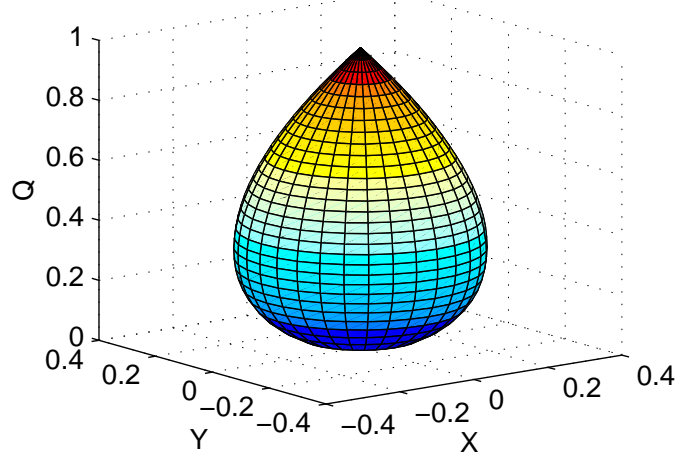

THREE-WAVE SURFACE, J=0.2

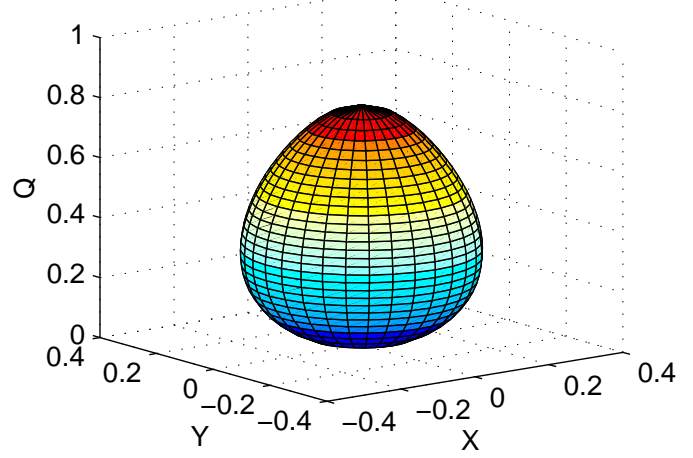

THREE-WAVE SURFACE, $\mathrm{J}=0.1$

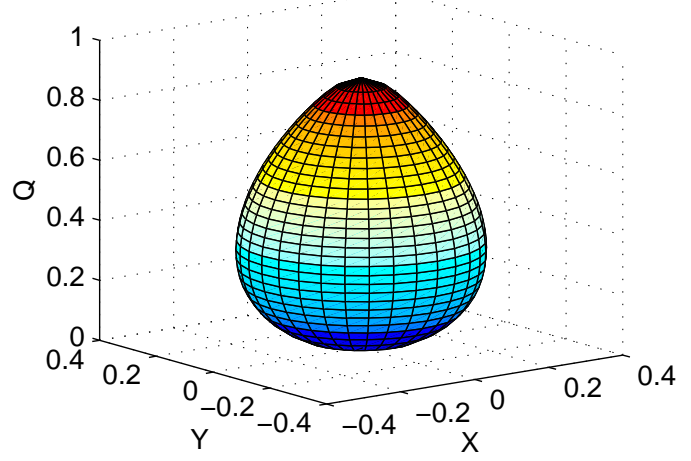

THREE-WAVE SURFACE, J $=0.3$

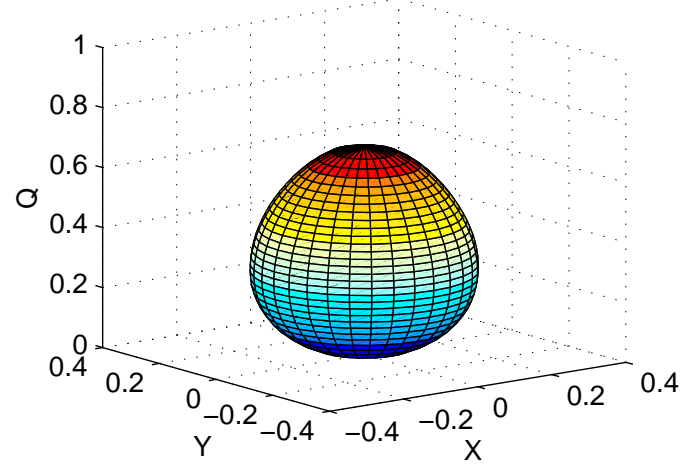

Figure 6. Surfaces of revolution about the $\mathcal{Q}$-axis, for $\mathcal{J} \in\{0.0,0.1,0.2,0.3\}$. The radius for given $\mathcal{Q}$ is given by the square-root of the cubic $-\mathcal{V}(\mathcal{Q})$. For given $\mathcal{J}$, the motion takes place on the intersection of the corresponding surface with a plane of constant $X$. 

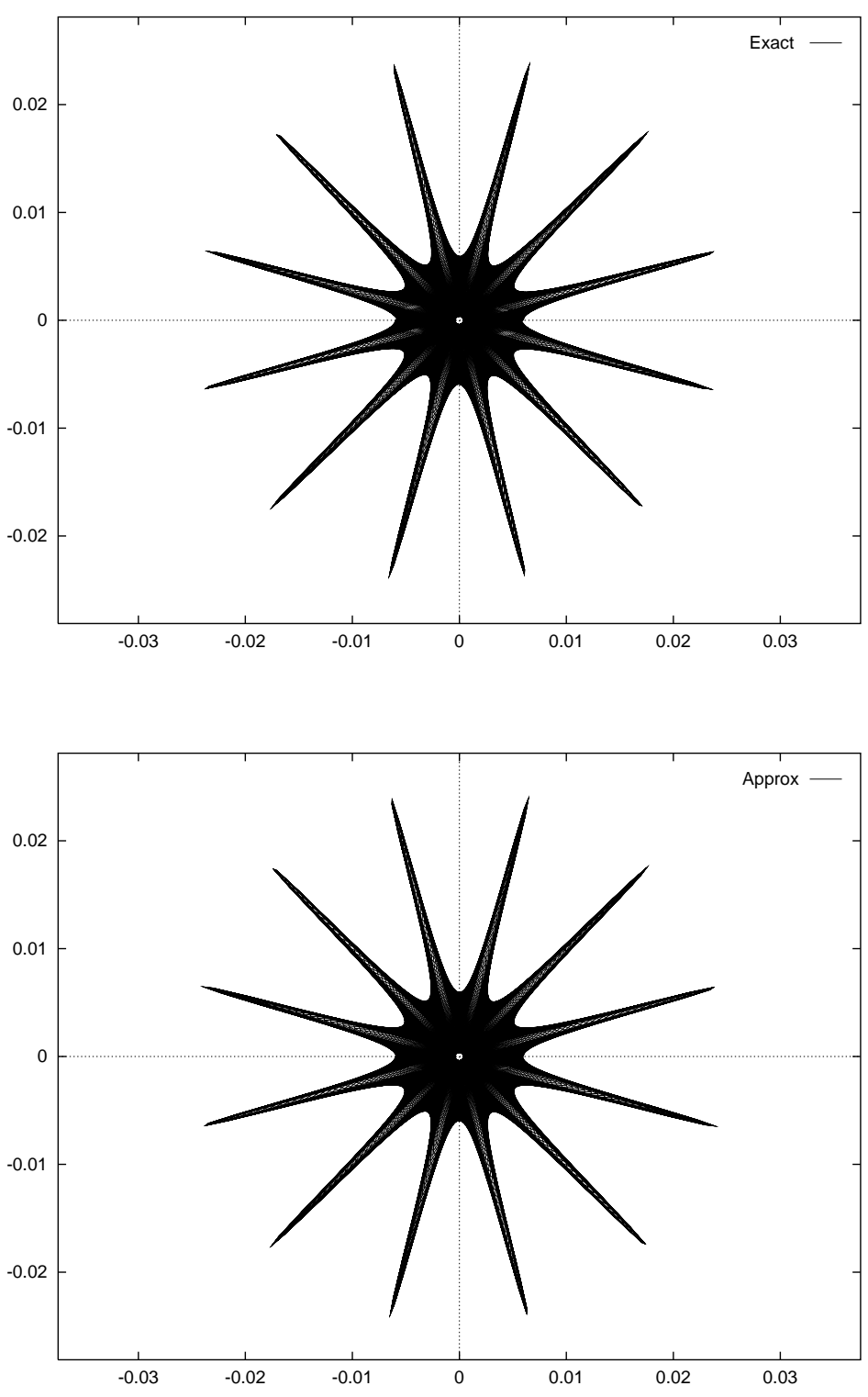

Figure 7. Horizontal projection of the solution for an integration of 1000 seconds. Top: solution of the 'exact' equations. Bottom: solution of the 'approximate' equations. 

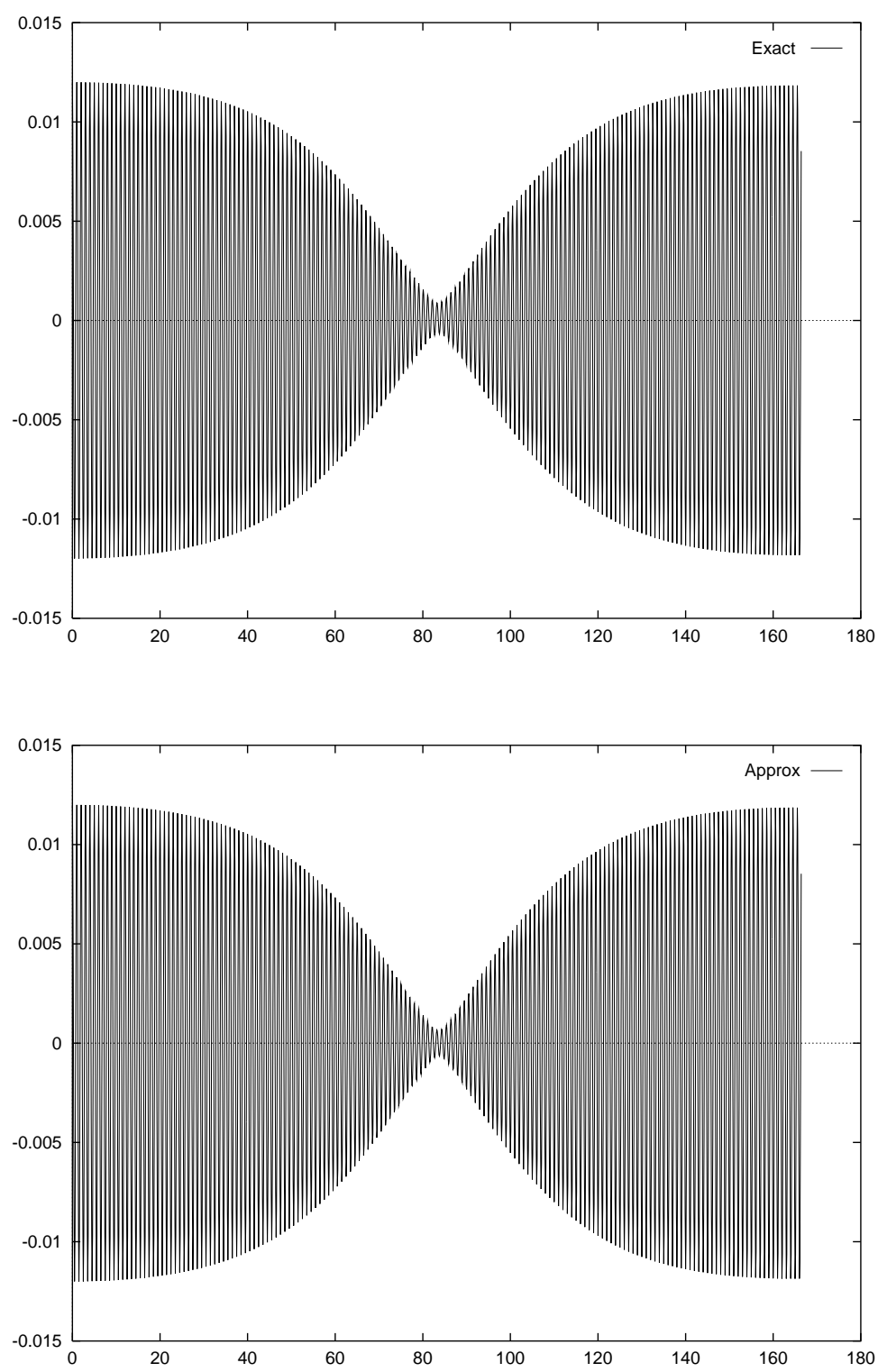

Figure 8. Vertical amplitude of the solution for the first modulation cycle (first 167 seconds). Top: solution of the 'exact' equations. Bottom: solution of the 'approximate' equations. 

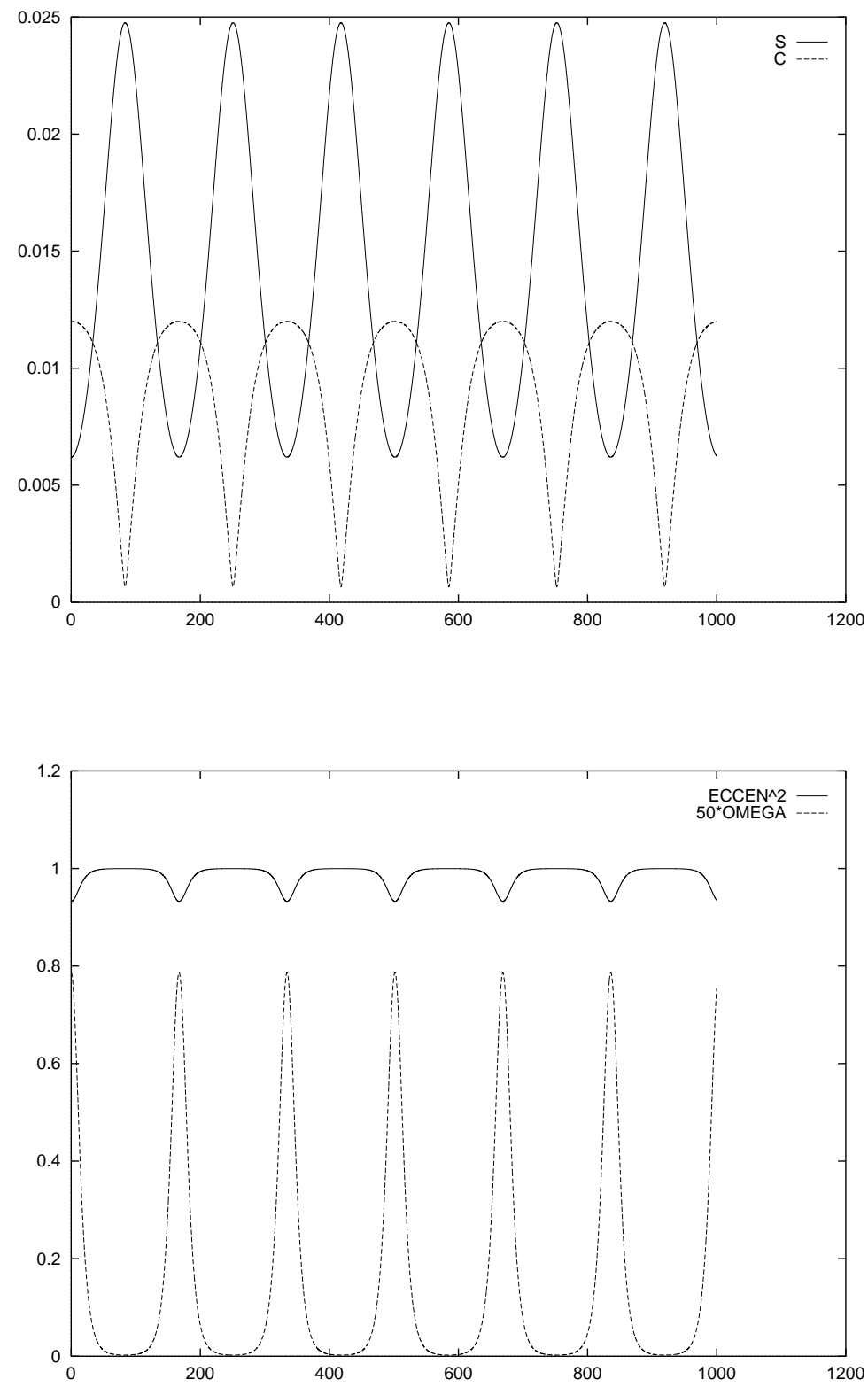

Figure 9. Top panel: Envelope amplitude of the approximate solution. $S=\sqrt{|a|^{2}+|b|^{2}}$ (solid line) and $C=|c|$ (dashed line). Bottom panel: Square of the eccentricity (solid line) and angular velocity $\Omega$ (scaled by 50) of the instantaneous ellipse (dashed line). 


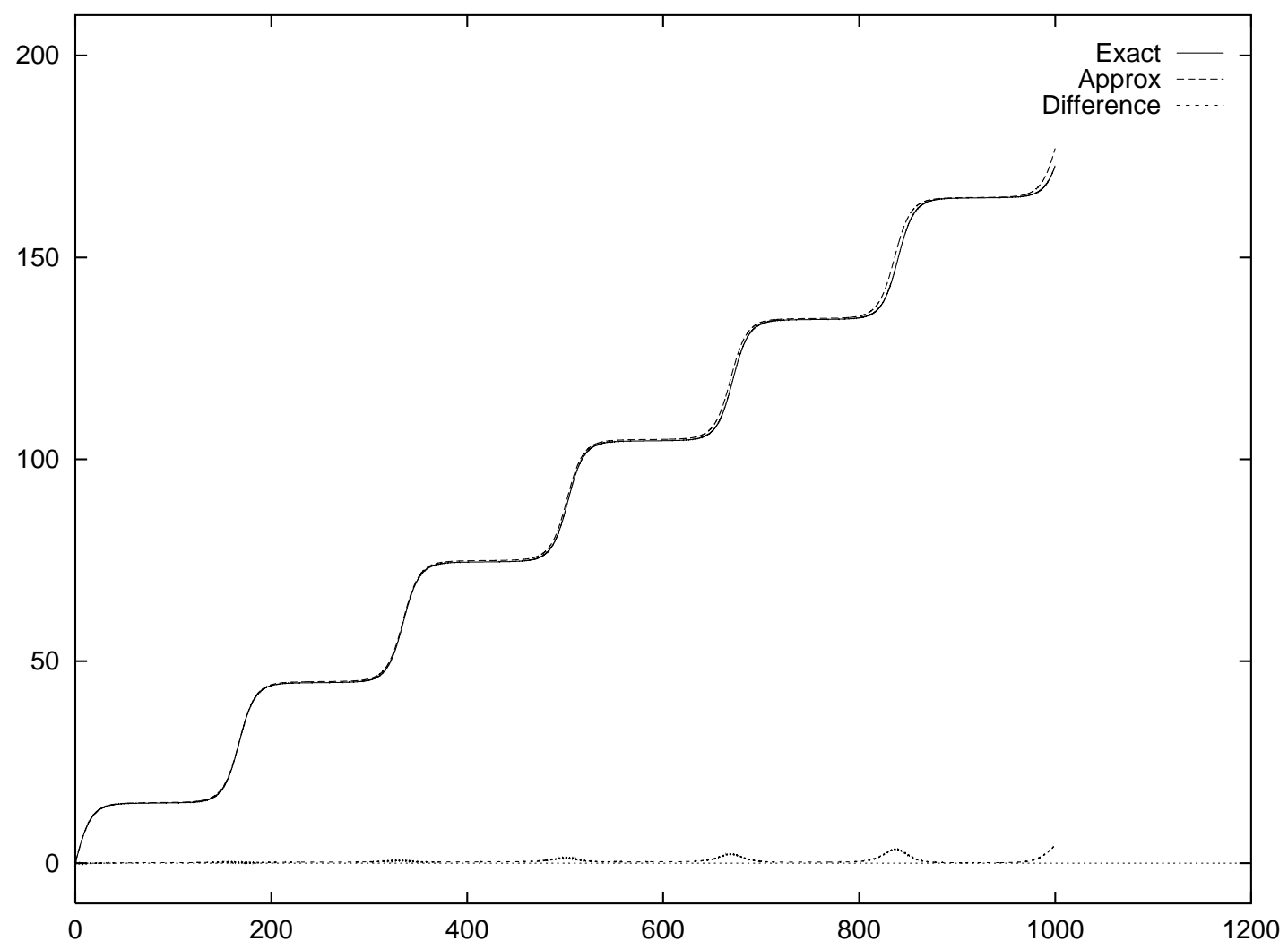

Figure 10. Azimuth angle (in degrees) for the 'exact' solution ( $\vartheta$, solid line) and the 'approximate' solution $(\theta$, dashed line). The difference $\theta-\vartheta$ is plotted as a dotted line. The azimuth $\Theta$ resulting from integration of (4.18) (not plotted) is indistinguishable from the values $\theta$ of the approximate solution. 